\section{Lentes intraoculares fáquicas de cámara posterior. Estudio comparativo entre los modelos PRL e ICL. Criterios de elección}

\section{Posterior chamber phakic intraocular lenses: a comparative study between ICL and PRL models. Choosing/selection criteria}

El objetivo de la presente es establecer comparativamente las similitudes y diferencias entre las dos lentes fáquicas precristalinianas de cámara posterior existentes para la corrección de la miopía e hipermetropía ICL (Staar) y PRL (IoltechZeiss/CibaVision)), en función de la información facilitada por las empresas productoras como de la revisión en PubMed de la literatura, y resumidas en la tablas I y II.

Las similitudes entre ambas más relevantes son: 1) Conveniencia de dos iridotomías profilácticas. Deben ser amplias y separadas $90^{\circ}$ para evitar el posible bloqueo pupilar y consecuente glaucoma agudo, especialmente en los hipermétropes. 2) Diámetro de zona óptica. Similar, ligeramente menor en la PRL, entre 4,5 y 5,5 mm dependiendo de la potencia de la lente, por lo que es predictible una porcentaje de halos luminosos y deslumbramientos nocturnos similares. 3) Elección del tamaño de la lente. Es también similar, añadiendo $0,5 \mathrm{~mm}$ al diámetro corneal blanco-blanco. 4) Cálculo biométrico a través de ellas. No alterado, es decir una vez implantada ninguna artefacta la medida del eje axial del globo ocular; y esto es especialmente relevante para el posterior cálculo de lente intraocular en caso de una posterior cirugía de catarata, cuya incidencia a largo plazo podría estar incrementada $(1,2) .5$ ) Implante secundario. Posibilidad en ambas de ser utilizadas como implante secundario en pacientes pseudofáquicos para corrección de ametropías residuales. 6) Rango de potencias comerciales similares. Aunque como luego analizaremos sea muy diferente su rango de corrección de dioptrías del paciente. En miopía desde -3 a -20 en PRL y desde -3 a -23 en ICL. En hipermetropía desde +2 a + 15 en PRL y de +3 a $+21,5$ en ICL. 7) Índice de refracción muy parecido. 1,46 en PRL, 1,45 en ICL.

8) Coste similar. Aproximadamente 800 euros.

Las diferencias entre ambas más relevantes son:

1) Tamaño. Sólo una lente hipermetrópica PRL de $10,6 \mathrm{~mm}$, frente a 4 tamaños en la ICL desde 11,0 a $12,5 \mathrm{~mm}$. Dos lentes miópicas PRL de 10,8 y 11,3 $\mathrm{mm}$, frente a 4 tamaños en la ICL desde 11,5 a 13.0 mm. 2) Posibilidad de corrección de astigmatismo miópico. De 1 a 6 dioptrías (con el eje situado en potencias positivas), exclusivamente con la lente ICL. 3) Material diferente. Silicona purificada hidrofóbica en las PRL frente a colágeno copolímero en las ICL. 4) Diferente técnica inyección. A través de microincisión de 3,2 $\mathrm{mm}$, se utilizan las pinzas para las lentes PRL, y el inyector para las ICL. 5) Distancia entre el cristalino y la lente fáquica. Mucho mayor en la zona central de la lente ICL que de la PRL, pero mucho menor y por tanto mayor posibilidad de contacto cataratógeno con el cristalino en la periferia de la lente ICL que de la PRL. 6)

Tabla I. Similitudes y diferencias entre las lentes fáquicas de cámara posterior

\begin{tabular}{lcc}
\hline & ICL & PR \\
\hline Tamaños en hipermetropía & $11,011,512,012,5$ & 10,6 \\
Tamaños en miopía & $11,512,012,513,0$ & $11,3-10,8$ \\
Corrección de astigmatismo & De 1 a 6 dioptrías & No \\
Rango comercial en miopía & -3 a -23 & -3 a -20 \\
Rango comercial en hipermetropía & +3 a $+21,5$ & +3 a +15 \\
Zona óptica & $4,65-5,5$ & $4,5-5,0$ \\
Material & Colágeno copolímero & Silicona purificada \\
Índice de refracción & 1,45 & 1,46 \\
Aconsejable ampliar incisión & No & Sí \\
Método inyección & Inyector (cartucho) & Pinza \\
Distancia central a cristalino & Mayor & Menor \\
Distancia a cristalino en periferia & Menor & Mayor \\
Apoyo periférico & Sulcus & Zónula \\
Empresa & Staar & Ioltech-Zeiss/Ciba V \\
Distribuidor nacional & Bloss Group & Imex \\
\hline \hline
\end{tabular}


Punto de apoyo. Comprobado por biomicroscopía ultrasónica, la mayoría de las lentes ICL apoyan en sulcus y la mayoría de las lentes PRL sobre la zónula. Esto explica que sólo se hayan publicado casos ocasionales de luxación a vítreo de la PRL y no de la ICL, desde dos meses hasta dos años después de su implantación (3-5).

Lo más importante desde el punto de vista clínico son dos hechos prácticos (tabla II y fig. 1): 1) Las lentes ICL/ PRL de potencia similar corrigen muy diferente número de dioptrías. Variando la potencia de la lente para un mismo caso según sea PRL o ICL una diferencia entre 1 a 9 dioptrías dependiendo del número de dioptrías a corregir, y del tipo de defecto. Además, dicha diferencia se incrementa a medida que aumenta el número de dioptrías a corregir. Así por ejemplo, para corregir -6 dioptrías de miopía hay que implantar una lente PRL de -5 pero ICL de $-8,5$. Para corregir -10 dioptrías de miopía, la PRL a implantar es de -8 y la ICL de -13,5; y para corregir una miopía de -18, la PRL es de -14 y la ICL de -22. En hipermetropía, para corregir +2 dioptrías hay que implantar una PRL de +2 o una ICL de $+3,20$. Y para corregir +6, la PRL a implantar es de +6 y la ICL +10; y para corregir un +11 deberá utilizarse una PRL de +12 pero una ICL de +21 . 2) Determinados defectos refractivos sólo pueden ser corregidas con una determinada lente ICL o PRL sin opción de elegir. Para pacientes miopes de -2 a $-3,25$ sólo es posible colocar una lente ICL, no existiendo alternativa en lente PRL. Este hecho es importante en casos postlasik de cirugía refractiva en los que no sea posible realizar retratamientos del defecto residual con láser (cornea plana o fina), o ante regresiones

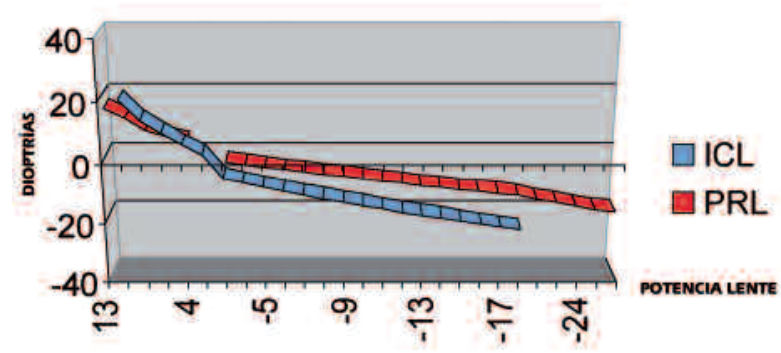

Fig. 1: Gráfico diferencial de lentes ICL y PRL para la misma refracción.

moderadas, o bien en casos en los que como primera opción refractiva se opte por cirugía intraocular (queratoconos fustre, etc.). Idéntica situación ocurre en miopes de más de -22, donde exclusivamente disponemos de lentes PRL para corregir tal defecto refractivo; y en pacientes hipermétropes de +11 a +13 donde solo es posible implantar una lente PRL.

Consideramos todo lo anteriormente comentado de interés para elegir la mejor opción refractiva en aquellos casos que precisen cirugía fáquica intraocular.

Díaz-Llopis $\mathrm{M}^{1}$, Montero $\mathrm{J}^{2}$, Amselem L ${ }^{2}$, Udaondo $\mathrm{P}^{2}$, García-Delpech $\mathrm{S}^{1}$

${ }^{1}$ Doctor en Medicina. Hospital General Universitario de Valencia. Valencia. Universidad de Valencia. Valencia. España

2 Doctor en Medicina. Hospital General Universitario de Valencia. Valencia. España E-mail: manuel.diaz@uv.es

Tabla II. Equivalencias entre las lentes fáquicas de cámara posterior ICL \& PRL en función de la refracción del paciente a corregir (*)

\begin{tabular}{|c|c|c|c|c|c|c|c|c|c|c|c|c|}
\hline \multicolumn{13}{|c|}{ Equivalente esférico refractivo en hipermetropía } \\
\hline & & $+2,00$ & & $+4,00$ & & $+6,00$ & & +8 & & +11 & & $+13,00$ \\
\hline Potencia ICL & & $+3,20$ & & $+6,64$ & & $+10,36$ & & +14 , & & +21 & ,08 & - \\
\hline Potencia PRL & & $+2,00$ & & $+4,00$ & & $+6,00$ & & +8 & & +12 & 00 & $+15,00$ \\
\hline Diferencia ICL y PRL & & 1,20 & & 2,64 & & 4,36 & & 6 & 38 & & 08 & - \\
\hline \multicolumn{13}{|c|}{ Equivalente esférico refractivo en miopía } \\
\hline & $-2,00$ & $-3,00$ & $-4,00$ & $-6,00$ & $-8,00$ & $-10,00$ & $-12,00$ & $-16,00$ & $-19,00$ & $-20,00$ & $-22,00$ & $-24,00-27,00$ \\
\hline Potencia ICL & $-3,12$ & $-4,59$ & $-6,02$ & $-8,74$ & $-11,28$ & $-13,66$ & $-15,90$ & $-19,99$ & $-22,78$ & - & - & $-\quad-$ \\
\hline Potencia PRL & - & - & $-3,56$ & $-5,23$ & $-6,82$ & $-8,36$ & $-9,84$ & $-11,27$ & $-14,65$ & $-15,30$ & $-16,57$ & $-17,81-19,62$ \\
\hline Diferencia ICL y PRL & - & - & 2,46 & 3,51 & 4,46 & 5,30 & 6,07 & 7,34 & 8,13 & - & - & $-\quad-$ \\
\hline
\end{tabular}

* Establecido para un cálculo teórico con dioptrías corneales medias Ks 42.5, profundidad de cámara anterior 3 mm y paquimetría corneal 560 micras. 


\section{BIBLIOGRAFÍA}

1. Pitault G, Leboeuf C, Leroux les Jardins S, Auclin F, Chong-Sit D, Baudouin C. Optical biometry of eyes corrected by phakic intraocular lenses. J Fr Ophtalmol 2005; 28: 1052-1057.

2. Menezo JL, Peris-Martínez C, Cisneros-Lanuza AL, Martínez-Costa R. Rate of cataract formation in 343 highly myopic eyes after implantation of three types of phakic intraocular lenses. J Refract Surg 2004; 20: 317-324.

3. García-Feijoó J, Hernández-Matamoros JL, CastilloGómez A, Lázaro C, Méndez-Hernández C, Martín T, et al. High-frequency ultrasound biomicroscopy of silicone posterior chamber phakic intraocular lens for hyperopia. J Cataract Refract Surg 2003; 29: 19401946.

4. Pitault G, Leboeuf C, Leroux Les Jardins S, Auclin F, Chong-Sit D, Baudouin C. Ultrasound biomicroscopy of posterior chamber phakic intraocular lenses: a comparative study between ICL and PRL models. J Fr Ophtalmol 2005; 28: 914-923.

5. Hoyos JE, Cigales M, Hoyos-Chacón J. Zonular dehiscence two years after phakic refractive lens $(P R L)$ implantation. J Refract Surg 2005; 21: 13-17. 